\title{
Artificial Rearing of Rat Pups Reveals the Beneficial Effects of Mother Care on Neonatal Inflammation and Adult Sensitivity to Pain
}

\author{
CYNTHIA B. DE MEDEIROS, ALISON S. FLEMING, CELESTE C. JOHNSTON, AND CLAIRE-DOMINIQUE WALKER
}

\begin{abstract}
Department of Psychology [C.B.D., A.S.F.], University of Toronto at Mississauga, Mississauga, Ontario L5L 1C6, Canada; School of Nursing [C.C.J., C.-D.W.], McGill University, Montreal H3A 2A7, Canada; Department of Psychiatry [C.B.D., C.-D.W.], McGill University, Montreal, H4H 1R3 Canada; Neuroscience Division [C.B.D., C.-D.W.], Douglas Institute in Mental Health, Montreal, H4H 1R3 Canada
\end{abstract}

\begin{abstract}
Repeated pain during brain development can have long-term consequences in both humans and animals. We previously showed that maternal care provided to pups experiencing pain reduced adult pain sensitivity. This study tested whether sensory stimulation was responsible for this effect. Rat pups were either mother-reared controls (MR-CON) or artificially reared (AR) with minimal (AR-MIN) or maximal (AR-MAX) stimulation provided daily. In each rearing condition, pups were either uninjected or injected from postnatal day (PND) 4 to 14 with saline $(0.9 \%)$ or formalin $(0.2-0.4 \%)$. Pain behavior and paw inflammation were scored. Thermal sensitivity and responses to formalin were tested in adulthood (PND 70). AR neonates, irrespective of sensory stimulation received, exhibited a pain response $(p<0.001)$, even with a mild formalin dose. Maternal rearing reduced inflammation during the second week of life compared with AR pups $(p<0.05)$. Early pain exposure did not modify adult pain sensitivity. However, rearing altered adult pain sensitivity such that uninjected MR-CON rats had lower pain sensitivities than uninjected AR rats $(p<0.05)$. This suggests that the beneficial effects of maternal rearing can be obliterated if additional stimulation/stress occurs during the early neonatal period. In addition, this suggests that optimal level of maternal stimulation exists that determines adult pain sensitivity. (Pediatr Res 66: 272-277, 2009)
\end{abstract}

$\mathrm{M}$ aternal care has numerous beneficial effects on attention, emotionality, and stress responsiveness in offspring $(1,2)$. In particular, maternal presence can reduce pain sensitivity in neonatal rats (3) and have long-term consequences in adult offspring (4). In humans, skin-to-skin contact, or kangaroo care, between mother and infant, has been demonstrated to soothe preterm infants in neonatal intensive care units (NICU) during painful treatments $(5,6)$. Thus, understanding the critical components of maternal care that are comforting is important for managing pain in preterm infants. In rats, maternal care occurs in a repertoire of behaviors including retrieving, licking, grooming, and crouching (7). Natural variations in the amount of licking and grooming provided to pups are a critical component of maternal care affecting development $(8,9)$. In particular, we demonstrated that offspring from high licking

Received March 3, 2009; accepted May 19, 2009

Correspondence: Claire-Dominique Walker, $\mathrm{PhD}$, Douglas Mental Health University Institute, 6875, Lasalle Blvd, Verdun, PQ H4H 1R3, Canada; e-mail: waldom@ douglas.mcgill.ca

Supported by a grant from the Canadian Institute for Health Research [C.C.J. and C.D.W. (MOP\#64307)] and the Fonds de la Recherche en Santé du Quebec (FRSQ). and grooming mothers displayed lower thermal pain sensitivity compared with animals from low licking mothers, suggesting that tactile stimulation might be an important component mediating the maternal effect on adult pain sensitivity in rats (4). To examine the role of tactile stimulation on pup development, we used the artificial rearing (AR) paradigm, which allows control over different aspects of maternal stimulation and nest environment (10-12). Dams lick the body and anogenital region of pups, serving thermoregulatory and excremental functions. In AR, this is mimicked with a paintbrush $(11,13,14)$. Experiments manipulating the amount of tactile stimulation received by offspring have shown that this essential component of maternal care influences development of attention (15), maternal behavior (13), and social skills (16). However, it is unknown whether tactile stimulation would modify pain perception in neonates and into adulthood. One aim of this study was to address this question using AR with varying levels of sensory stimulation.

Many adult behavioral features can be greatly influenced by early experience and perinatal history in both humans and animals. In particular, preterm human neonates exposed to repeated noxious events can have behavioral changes lasting until young adulthood (17-19). Because early stressors can modify the effect of sensory stimulation in AR animals (15), we determined whether concomitant application of repeated modest pain during the neonatal period in pups reared with or without mothers would alter pain sensitivity in adulthood. We chose to repeatedly administer low formalin doses during the first 2 weeks of life to best approximate the repeated inflammatory pain preterm infants experience in the NICU. The main objectives of this study were to determine whether i) the type and amount of sensory stimulation received by AR pups differentially affects growth, inflammation, and pain behavior compared with mother-reared pups and ii) maternal care has a long-lasting impact on adult pain thresholds in animals receiving different levels of pain early in life.

Abbreviations: AR, artificially reared; AR-MAX, artificially reared maximal stimulation; AR-MIN, artificially reared minimal stimulation; FOR, formalin; MR, maternally reared; MR-CON, maternally reared control; PND, postnatal day; SAL, saline; UI, uninjected 


\section{MATERIALS AND METHODS}

Animals. Rats were born and raised at the University of Toronto at Mississauga from a stock originally from Charles River Farms (St. Constant Quebec, Canada). Animals were maintained under controlled lighting (12:12 $\mathrm{h}$ light:dark cycle, lights on at $0800 \mathrm{~h})$, humidity $(50-60 \%)$, and temperature $\left(22^{\circ} \mathrm{C}\right)$ with food and water available ad libitum. Ten (60- to 90-d old) primiparous female Sprague Dawley rats were mated with sexually experienced males. On the day after birth, postnatal day (PND) 1, litters were culled to 10 males and 4 females, when possible. On PND 3, six males were removed from each litter and randomly distributed into two AR conditions. The four remaining male pups served as maternally reared (MR) controls (MR-CON). A total of 75 male rats were used, and the results of two cohorts were pooled. All the procedures were approved by the Animal Care Ethics Committees at the University of Toronto and McGill University and followed guidelines from the Canadian Council on Animal Care.

Surgery and artificial rearing. Pups were weighed and a topical anesthetic (EMLA) was applied to the cheek. A lead wire (stainless steel, $0.25 \mathrm{~mm}$ diameter) covered with polyethylene (PE) 10 tubing was used to pierce the cheek. The PE 10 tubing was reinforced with washers and glued into place. After the cheek cannulation, each AR pup was individually placed in a plastic cup (11 cm diameter $\times 15 \mathrm{~cm}$ deep) with corncob bedding (Bed O'Cobs). The cups were placed in a water-filled tank with regulated temperature $\left(34-37^{\circ} \mathrm{C}\right)$. Pups were connected by the cheek cannula to a time-controlled infusion pump (Harvard Apparatus Syringe, PHD 2000), which administered an artificial milk diet (20) daily for 10 min every hour. On PND 3, the volume of milk infused was equal to $34 \%$ of the mean body weight per pump and increased by $2 \%$ per day. Every morning, pups were disconnected from the pumps and weighed. Cheek cannulas were flushed and new syringes were filled with fresh milk. In one cohort, a group of pups underwent surgery, and the cannula was removed before pups were returned to the mother (MR-SHAM). This group served to control for the effects of surgery. MR-SHAM animals (saline injected, $n=2$; formalin-injected, $n=8$ ) did not differ from MR-CON animals on all the parameters tested; thus the results were omitted for clarity of presentation.

Groups and treatments. AR rats were randomly assigned to either the artificially reared minimal stimulation (AR-MIN) or artificially reared maximal stimulation (AR-MAX) group. AR-MIN animals received two 30-s anogenital stimulations daily using a wet camelhair paintbrush (morning and evening). This was done to stimulate urination and defecation and to limit sensory stimulation. AR-MAX animals received the same anogenital stimulations with five additional 11/2-minute body stimulations (whole body stimulation excluding the anogenital region) with a dry camelhair paintbrush. A minimum of $1 \mathrm{~h}$ occurred between body stimulations. Stimulations occurred daily from PND 3 to 16 . However, on PND 3, only three body stimulations and one anogenital stimulation were given due to the surgical procedure. On PND 18, AR pups were removed from the pumps and weaned onto a mixture of artificial milk and powdered chow. On PND 22, both AR and MR rats were weighed and pair housed with a same sex conspecific. Rats remained undisturbed until adult testing

Early pain treatment. Animals in the AR and MR-CON rearing conditions were further randomly assigned to three early life treatment groups: i) no injection (UI), ii) saline injection (SAL) or iii) formalin injection (FOR) for a total of nine experimental groups: AR-MIN-FOR $(n=9)$, AR-MIN-SAL $(n=8)$, AR-MIN-UI $(n=6)$, AR-MAX-FOR $(n=7)$, AR-MAX-SAL $(n=$ 9), AR-MAX-UI $(n=8)$, MR-CON-FOR $(n=10)$, MR-CON-SAL $(n=9)$, and MR-CON-UI $(n=9)$. Formalin or saline injections $(10 \mu \mathrm{L})$ were administered in the footpad of the hindpaw twice daily (morning and evening) between PND 4 and 14. Formalin doses were $0.2 \%$ from PND 4 to 8 and $0.4 \%$ from PND 9 to 14. The injected paw was alternated daily as previously described (21). AR animals were disconnected from the pumps and brought into the injection room. AR-MIN animals received an injection and were placed back into the cups without additional stimulation. To simulate maternal care, AR-MAX animals received an anogenital stimulation and a body stimulation immediately following morning injections and only a body stimulation after evening injections. Pain behavior of AR injected pups was observed and scored every $3 \mathrm{~min}$ for $15 \mathrm{~min}$ after each injection. Pups were reconnected to the pump following observations. The pain scoring scale was as follows: $0=$ normal, $1=$ favor, $2=$ lifting paw, $3=$ lick/shake/flinch the paw, 4 = paw in mouth. MR animals were brought into the injection room with the dam and litter, injected, and immediately placed back in the nest. Since mothers typically hover rapidly after pup treatment, it was not possible to unequivocally score pain behavior of MR-CON pups.

We recorded daily paw inflammation, before morning injections, using the following inflammation rating scale: $0=$ normal, $1=$ red or swollen paw, $2=$ inflamed paw (red and swollen). Both left and right hind paws were scored from PND 5 to 14
Determination of thermal sensitivity using Hargreaves test. From PND 66 to 69 , all animals were habituated to the Hargreaves testing chambers (six boxes, $11 \times 5 \times 20 \mathrm{~cm}$; IITC Life Sciences Inc, Woodland Hills, CA) for 5-min each day. Between PND 70 and 74, rats were tested for thermal sensitivity using the Hargreaves test (22). Animals were allowed to acclimate for $5 \mathrm{~min}$ before testing. Light beam intensity was set to $30 \%$ for higher sensitivity (4) and room temperature was maintained at $22^{\circ} \mathrm{C}$. The light beam was focused and alternated between left (three trials) and right (three trials) hind paws across six trials. Latency to lift paw was measured as the time between the start of the light beam until the animal lifted its paw. Animals were returned to their home cage after testing.

Inflammatory pain. Between PND 77 and 87, behavioral pain response to formalin was tested. Four rats were tested concurrently and randomly in each trial. Animals were s.c. injected $(10 \mu \mathrm{L})$ in the plantar surface of the hind paw with either formalin $(2.5 \%)$ or saline $(0.9 \%)$. The solution and injected paw were counterbalanced across two test days separated by an 8 - to 10-d interval. Immediately after the injection, animals were placed in individual Plexiglas chambers $(30 \times 15 \times 30 \mathrm{~cm})$ set on a glass plate above a mirror for observations. A program provided by Dr. F. Abbott (Department of Psychiatry, McGill University) was used to score three behavioral categories: i) pain (normal, favor, lift, and lick/shake/flinch), ii) behavioral activity (sleep, groom, locomotion, etc.), and iii) physiological state (normal, piloerection, and sickness) (21). Behavior was scored every minute for $60 \mathrm{~min}$. In one of the cohorts, two observers (one blind to neonatal treatment) scored behavior independently to establish interrater reliability, which was above $88 \%$.

Statistical analysis. All results are expressed as means \pm SEM. Results were analyzed by two- or three-way analysis of variance (ANOVA) depending on the experimental design and using neonatal treatment group (UI, SAL, FOR), rearing condition (AR-MIN, AR-MAX, MR-CON), and repeated age intervals as factors. When significant interactions were found, they were separated into simple main effects. Post hoc pairwise contrasts were conducted using Tukey's honestly significant differences test. Significance level was set at $p<0.05$.

\section{RESULTS}

Pup growth and effect of rearing condition. At the time of cannulation, all pups had similar body weights. Regardless of treatment, body weight gain was slower in AR pups compared with MR pups from PND 4 to 14 (Fig. 1). In each rearing condition, a two-way ANOVA confirmed that there was no significant effect of treatment (SAL, FOR, or UI) or time $\times$

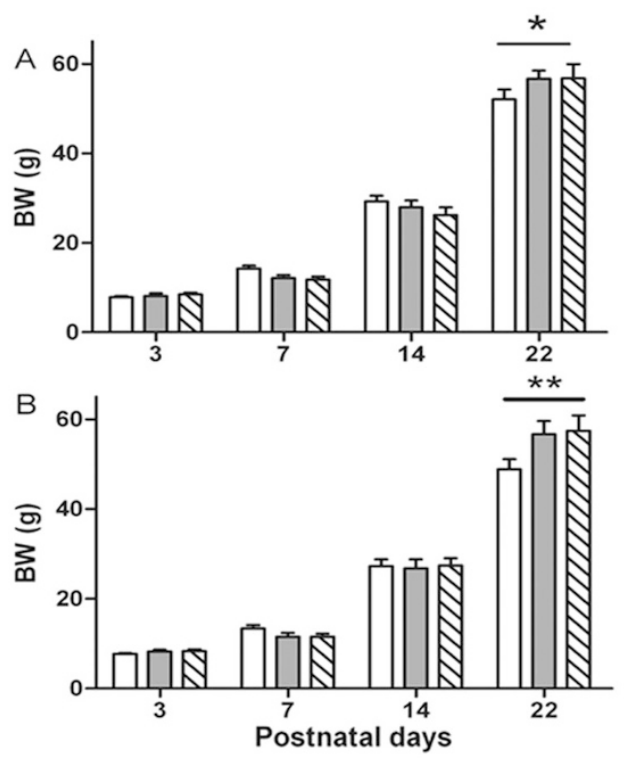

Figure 1. Effect of rearing condition on body weight $(\mathrm{g})$ in uninjected $(A)$ or formalin injected (B) pups of MR-CON (open bars), AR-MAX (gray bars), and AR-MIN (hatched bars) pups. Values represent the mean \pm SEM of $8-10$ animals/group. ${ }^{* *} p<0.01$ and $* p<0.05$ among rearing condition with AR-MIN and AR-MAX differing from MR-CON. 
treatment interaction on body weight. Rearing condition was a stronger predictor of body weight than treatment, because a significant interaction between rearing condition (AR-MIN, AR-MAX, MR-CON) and time $(p<0.001)$ was found. At weaning (PND 22), AR-MIN and AR-MAX pups weighed more than MR-CON pups ( $p<0.05$ or 0.01 ).

Pup pain responses to neonatal formalin injection. Pain scores were recorded for 15 min after injection but only in AR pups. MR pups were not observed because scores would be confounded by maternal behavior. Morning and evening pain scores did not differ between treatment and rearing conditions (data not shown). A low formalin dose $(0.2 \%$ from PND 4 to 8 and $0.4 \%$ from PND 9 to 14 ) significantly increased pain scores compared with saline at all ages (Fig. 2). A three-way ANOVA with repeated measures of pain scores across age showed significant effects of treatment (formalin, $p<0.001$ ) and age $(p<0.001)$ but not sensory stimulation intensity (AR-MIN versus AR-MAX, $p=0.226$ ). Cumulative pain scores increased with age, partly due to the expansion of the repertoire of behavioral responses with developmental age.

Effect of maternal care on paw inflammation. Both paws of all pups were scored for inflammation every morning. No significant inflammation $24 \mathrm{~h}$ after treatment was found in saline injected pups among all rearing conditions (data not shown). Redness and swelling occurred in paws of formalin treated animals $24 \mathrm{~h}$ after injection in all rearing conditions (Fig. 3), although inflammation decreased significantly with age $(p<0.001)$, even when the formalin dose increased (PND 9-14). A three-way ANOVA demonstrated a significant age effect $(p<0.001)$ and a near significant maternal rearing effect $(p=0.0761)$ on paw inflammation. MR-CON pups had a significantly lower inflammation score on postnatal days 9 , 11 , and $12(p<0.05)$ compared with both AR groups, indicating that the mother can reduce inflammation.

Effect of rearing condition on pain sensitivity of adult offspring. Offspring from all neonatal treatment groups and rearing conditions were tested in adulthood for sensitivity to thermal pain using the Hargreaves test and inflammatory response to formalin injection. In the Hargreaves test, no

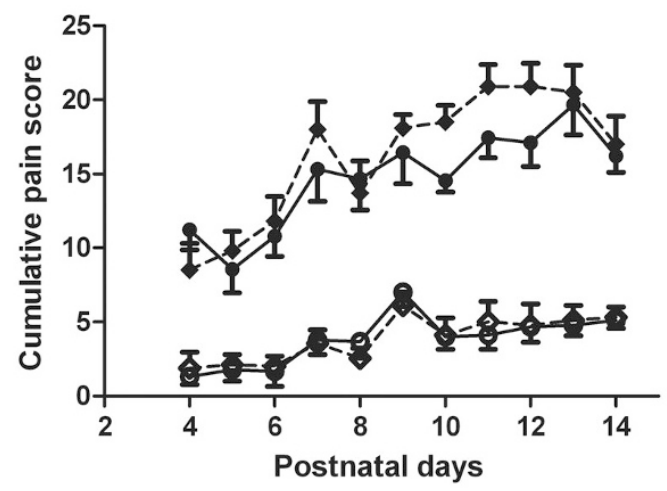

Figure 2. Cumulative pain scores in neonates treated with saline or formalin from PND4-14 in AR-MIN (SAL, open diamonds; FOR, closed diamonds) or AR-MAX (SAL, open circles; FOR, closed circles). Behavioral scoring occurred every $3 \mathrm{~min}$ for $15 \mathrm{~min}$ after injections. Treatment and age effects were significant $(p<0.001)$ but not rearing condition. Values represent the mean \pm SEM of $9-10$ animals/group.

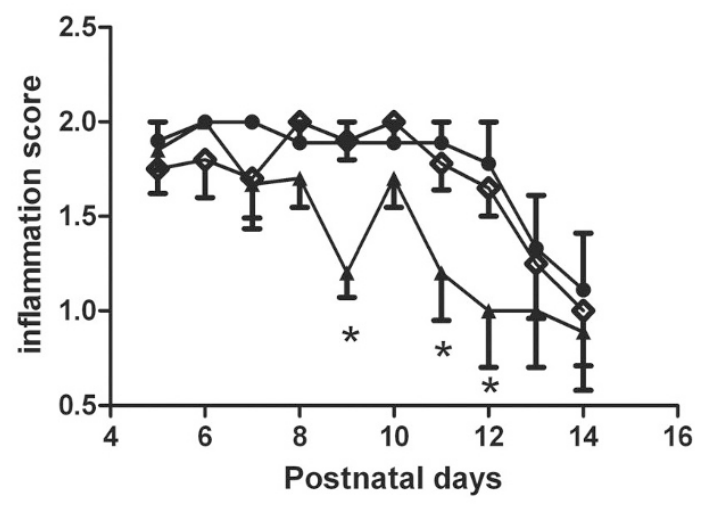

Figure 3. Age-related paw inflammation scores $24 \mathrm{~h}$ after formalin injections in pups from AR-MAX (closed circles), AR-MIN (open diamonds), or MR-CON (closed triangles). Age effect was significant $(p<0.001)$ and rearing condition effect was close to significant $(p=0.076)$. Values represent the mean \pm SEM of $9-10$ animals/group. ${ }^{*} p<0.05$ among rearing condition with AR-MIN and AR-MAX differing from MR-CON.

significant difference in paw withdrawal latency was found between left and right paws, thus measures were pooled for each animal. Among UI offspring, MR-CON animals displayed longer latencies than AR-MAX animals $(p=0.038$, Fig. $4 C$ ). For animals that received neonatal saline (Fig. $4 A$ ) or formalin (Fig. $4 B$ ) treatment, this difference was not observed suggesting that the rearing condition effect was abolished by early stimulation (handling and injection).

In adulthood, rats exhibited significantly greater pain responses when acutely injected with formalin compared with saline $(p<0.001)$ across neonatal treatments and rearing conditions. Pain behavior after the saline (Fig. $5 A$ ) and formalin (Fig. 5B) injection was scored every minute for $60 \mathrm{~min}$. Cumulative scores over 5-min intervals were calculated for offspring originating from the neonatal treatments (UI, SAL, FOR) and rearing conditions (AR-MIN, AR-MAX, MRCON). Rats from all groups showed the typical biphasic pain response to formalin injection with an initial phase from 1 to $8 \mathrm{~min}$ and a second, prolonged phase, from 9 to $60 \mathrm{~min}$ (data not shown). For the second phase, we computed an integrated value of the overall pain score by calculating the area under the curve (AUC) (Fig. 5B). A two-way ANOVA indicated a significant treatment effect $(F(2,70)=3.4, p=0.04)$ and a near significant effect of rearing condition $(p=0.068)$ on integrated pain scores. The interaction between neonatal treatment and rearing condition was not significant. For each subject in both AR groups, the AUC was expressed as a percentage of the value obtained for MR-CON offspring in each of the two cohorts pooled (Fig. 5C). A two-way ANOVA showed a main effect of rearing condition $(F(2,69)=3.24$, $p=0.046$ ), but no treatment effect or interaction between neonatal treatment and rearing condition. In all neonatal treatment groups, adult pain behavior in AR rats was higher than in MR-CON rats although the difference reached significance only in UI offspring $(p<0.05)$.

\section{DISCUSSION}

Evidence from human and animal literature suggests that maternal care is beneficial for reducing offspring pain sensi- 

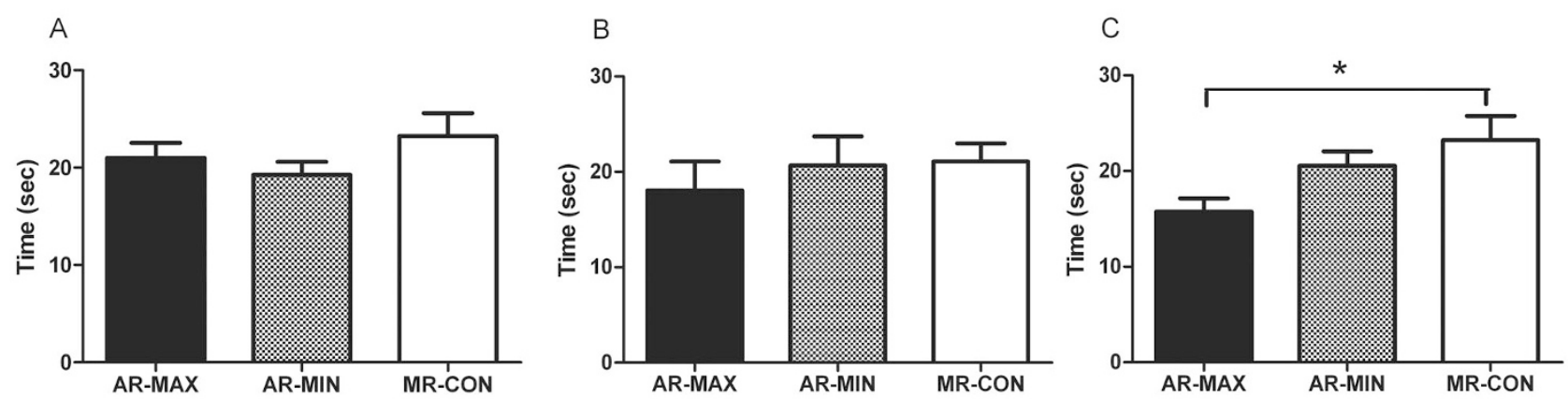

Figure 4. Latency to lift paw during Hargreaves testing in adult offspring that had received saline $(A)$, formalin $(B)$, or no injection $(C)$ from PND 4 to 14 . The rearing condition effect was significant $(p=0.038)$ among UI animals. Results are expressed as mean \pm SEM of $6-10$ animals/group. $* p<0.05$ compared with MR-CON
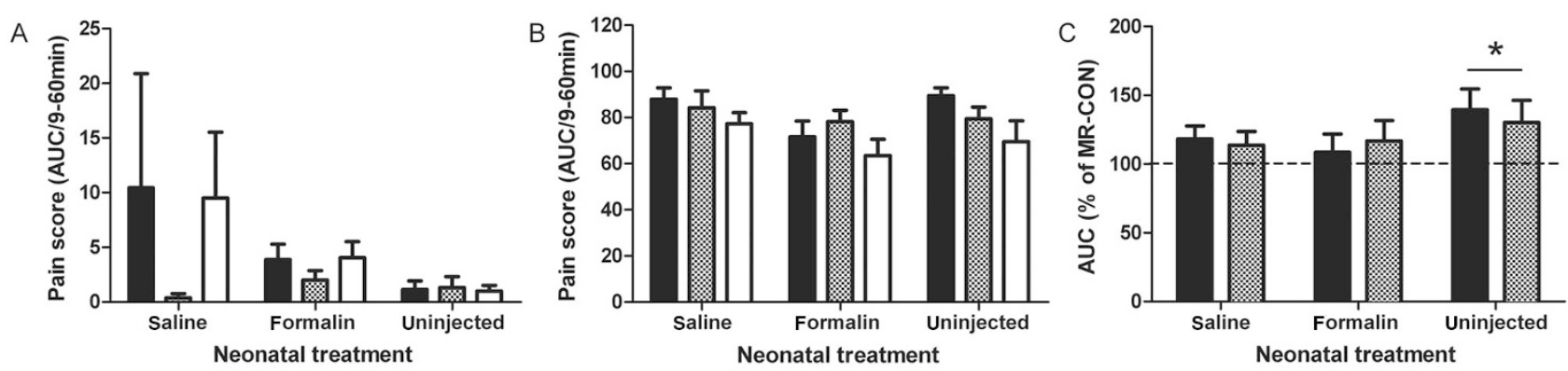

Figure 5. Behavioral pain response to saline or formalin (2.5\%) injections in adult offspring from AR-MAX (black bars), AR-MIN (gray bars), and MR-CON (open bars) receiving different neonatal treatments. Integrated pain response expressed as area under the curve (AUC) was calculated for the second response phase (9-60 min) in rats receiving saline $(A)$ or formalin $(B)$. Treatment effect was significant $(p=0.0397)$ and rearing effect was almost significant $(p=0.068)$. The integrated formalin pain response was expressed as a percentage of the MR-CON response in AR-MAX (black bars) and AR-MIN (gray bars) for each experimental series $(C)$. Rearing condition was significant $(p=0.046)$. Values represent mean \pm SEM of 6 to 9 animals/group. * $p<0.05$ among rearing condition with AR-MIN and AR-MAX differing from MR-CON.

tivity, whether pain exposure occurs during developmental periods or in adulthood. In a recent study on rats, we demonstrated that adult offspring from low licking and grooming mothers displayed higher thermal pain sensitivity than those from high licking and grooming mothers (4), suggesting that the amount of sensory stimulation the mother provides might be an important factor modulating later pain responsiveness. In this study, we investigated whether licking-like stimulation provided to AR pups would be sufficient to reduce long-term sensitivity to thermal and inflammatory pain. We also evaluated whether variation in the type and quantity of sensory stimulation provided to neonatal rat pups would modify the consequences of early pain exposure. Our results showed that in groups not exposed to the neonatal stress of injections, maternal rearing reduced sensitivity to both thermal and inflammatory pain. Moreover, contrary to our prediction, ARMAX rats exhibited higher pain sensitivity in adulthood than AR-MIN rats. Repeated neonatal injections, both saline and formalin, did not affect adult pain sensitivity to formalin in this study, which contrasts other studies using different models of early pain (23). However, exposure to neonatal stressors completely eliminated the effects of rearing on adult pain sensitivity although, overall, there was a trend for higher pain sensitivity in the AR groups.

The reduced pain sensitivity in MR compared with AR adult animals was observed after tests of thermal pain sensitivity or formalin injection, however, only in animals UI during the neonatal period. In UI animals, the AR-MAX group was more sensitive to pain compared with MR-CON and AR-MIN. The potentiating effect of high levels of tactile stimulation coupled with AR on pain sensitivity, although counter to our prediction, is not without precedence. Previous data suggests that an optimal amount of sensory input received by AR rats restores some, but not all, deficits due to AR. For instance, AR-MIN animals showed an enhanced startle response (15) and amphetamine-induced locomotion (11) compared with MR-CON rats. These deficits were eliminated in AR-MAX animals. However, in experiments coupling prenatal stress and AR, receiving an "optimal" level of sensory stimulation (AR-MAX) produced more locomotor activity than the AR-MIN condition (15), suggesting that more licking-like stimulation was too intense for these prenatally stressed animals. We suspect that a similar phenomenon is occurring in our UI group, although it is unclear why neither the saline nor the formalin groups, which were subjected to stress from injections and pain, did show the same responses to sensory stimulation. Our finding of greater negative effects of additional sensory stimulation (AR-MAX) on adult pain sensitivity is, however, consistent with human preterm infant studies, suggesting that specific types of sensory stimulation can have detrimental effects on physiologic and behavioral regulation (24).

Although some animals received a painful stimulus (formalin) early in development, we did not observe the development of pain sensitization to thermal or inflammatory pain. This 
contrasts with our earlier work, which documented sensitization of adult pain responses to formalin, but not thermal pain, in neonatal pups exposed to formalin (4). It is possible that we are at the threshold for detecting a reliable early formalin effect. If higher concentrations of formalin were administered neonatally, more consistent sensitization may be produced. To mimic the degree of repeated pain experienced by preterm infants subjected to multiple heel sticks daily in the NICU, we purposely chose a lower dose of formalin $(0.2-0.4 \%)$ than is typically used in neonatal rat experiments to induce strong pain and/or inflammation $(25,26)$. Despite using low formalin concentrations, we found that young animals injected with formalin, but not saline, exhibited significant behavioral pain responses during the treatment period. We also found that over time, pain scores intensified. The age-related increase in pain scores reflects the maturation of nociceptive pathways and receptor fields in neonates (27-29) and their expanding behavioral repertoire (30).

Although neonatal injections alone did not affect adult pain sensitivity, it is clear that they did modulate rearing condition effects. Maternal rearing did not significantly reduce adult pain sensitivity in the injected groups. It is possible that additional handling and arousal from the injection procedure may have obliterated any effects of licking-like stimulation provided immediately after injections to AR-MAX pups and those provided daily to both AR groups. As previously shown, mothers rapidly retrieve pups after an injection followed by licking and grooming before taking a nursing posture that reduces pup arousal. Even though the stimulation procedure was designed to mimic mother's sensory stimulation, we observed that AR pups maintained a higher degree of arousal for longer periods after injection compared with MR-CON counterparts. Whether AR pups adapted to a higher degree of stress and arousal allowing them to better cope with pain in adulthood requires further investigation.

Maternal rearing effects were seen during the neonatal period and adulthood. Inflammation levels after formalin injections were significantly reduced in MR compared with AR animals, regardless of the intensity of licking-like stimulation provided. This reduced inflammation, most significant during the second week of the injection period, might be attributed to increased maternal licking of the inflamed paw. Another possibility is that formalin-treated pups increased ultrasonic vocalizations to stimulate maternal care (31). Indeed, we demonstrated earlier that pups subjected to pain stimulate higher licking and grooming from their mothers (32). Littermates may also serve a beneficial role in reducing arousal and stimulating a more rapid return to baseline after painful procedures. AR studies on rats have demonstrated the beneficial role of littermates in reducing some of the negative consequences of being reared in isolation, such as those on social learning (16).

In conclusion, we have shown the beneficial effects of maternal care to reduce formalin-induced inflammation early in life and lowering pain sensitivity in adulthood, specifically in animals not exposed to neonatal stress. In addition, we have provided support for low formalin concentrations as an ade- quate experimental protocol to trigger significant pain responses in neonatal rats. This is important because lower doses may more realistically represent the repeated procedural pain experienced by preterm infants in the NICU. In rats, maternal care is important in establishing thermal pain thresholds (4) and in humans, kangaroo care, skin-to-skin contact between mother and infant, has been demonstrated to calm infants experiencing pain (6). Our study emphasizes the importance of maternal care during the neonatal period, although additional studies are required to determine which components of the mother care repertoire are critical in modulating long-term physiologic and behavioral responses of offspring.

Acknowledgments. We thank Ms. Zhifang Xu, Ms. Veronica Afonso, and Mr. Xavier D'Souza for help with testing and Ms. Hannah Oatley and Ms. Diana Medeiros for help with artificial rearing.

\section{REFERENCES}

1. Champagne DL, Bagot RC, van Hasselt F, Ramakers G, Meaney MJ, de Kloet ER, Joëls M, Krugers H 2008 Maternal care and hippocampal plasticity: evidence for experience-dependent structural plasticity, altered synaptic functioning, and differential responsiveness to glucocorticoids and stress. J Neurosci 28:6037-6045

2. Kaffman A, Meaney MJ 2007 Neurodevelopmental sequelae of postnatal maternal care in rodents: clinical and research implications of molecular insights. J Child Psychol Psychiatry 48:224-244

3. Blass EM, Shide DJ, Zaw-Mon C, Sorrentino J 1995 Mother as shield: differential effects of contact and nursing on pain responsivity in infant rats-evidence for nonopioid mediation. Behav Neurosci 109:342-353

4. Walker CD, Xu Z, Rochford J, Johnston CC 2008 Naturally occurring variations in maternal care modulate the effects of repeated neonatal pain on behavioral sensitivity to thermal pain in the adult offspring. Pain 140:167-176

5. Johnston CC, Stevens B, Pinelli J, Gibbins S, Filion F, Jack A, Steele S, Boyer K, Veilleux A 2003 Kangaroo care is effective in diminishing pain response in preterm neonates. Arch Pediatr Adolesc Med 157:1084-1088

6. Johnston CC, Filion F, Campbell-Yeo M, Goulet C, Bell L, McNaughton K, Byron J, Aita M, Finley GA, Walker CD 2008 Kangaroo mother care diminishes pain from heel lance in very preterm neonates: a crossover trial. BMC Pediatr 8:13

7. Parada M, King S, Li M, Fleming AS 2008 The roles of accumbal dopamine D1 and D2 receptors in maternal memory in rats. Behav Neurosci 122:368-376

8. Menard JL, Hakvoort RM 2007 Variations of maternal care alter offspring levels of behavioural defensiveness in adulthood: evidence for a threshold model. Behav Brain Res 176:302-313

9. Menard JL, Champagne DL, Meaney MJ 2004 Variations of maternal care differentially influence 'fear' reactivity and regional patterns of cFos immunoreactivity in response to the shock-probe burying test. Neuroscience 129:297-308

10. Dominguez HD, Thomas JD 2008 Artificial rearing. Methods Mol Biol 447:85-100

11. Lovic V, Fleming AS, Fletcher PJ 2006 Early life tactile stimulation changes adult rat responsiveness to amphetamine. Pharmacol Biochem Behav 84:497-503

12. West JR 1993 Use of pup in a cup model to study brain development. J Nutr 123:382-385

13. Gonzalez A, Fleming AS 2002 Artificial rearing causes changes in maternal behavior and c-fos expression in juvenile female rats. Behav Neurosci 116:999-1013

14. Gonzalez A, Lovic V, Ward GR, Wainwright PE, Fleming AS 2001 Intergenerational effects of complete maternal deprivation and replacement stimulation on maternal behavior and emotionality in female rats. Dev Psychobiol 38:11-32

15. Burton C, Lovic V, Fleming AS 2006 Early adversity alters attention and locomotion in adult Sprague-Dawley rats. Behav Neurosci 120:665-675

16. Melo AI, Lovic V, Gonzalez A, Madden M, Sinopoli K, Fleming AS 2006 Maternal and littermate deprivation disrupts maternal behavior and social-learning of food preference in adulthood: tactile stimulation, nest odor, and social rearing prevent these effects. Dev Psychobiol 48:209-219

17. Grunau R 2002 Early pain in preterm infants. A model of long-term effects [Review] Clin Perinatol 29:373-394

18. Grunau RE, Oberlander TF, Whitfield MF, Fitzgerald C, Morison SJ, Saul JP 2001 Pain reactivity in former extremely low birth weight infants at corrected age 8 months compared with term born controls. Infant Behav Dev 24:41-55

19. Wollgarten-Hadamek I, Hohmeister J, Demirakça S, Zohsel K, Flor H, Hermann C 2009 Do burn injuries during infancy affect pain and sensory sensitivity in later childhood? Pain 141:165-172

20. Messer M, Thoman EB, Galofre A, Dallman T, Dallman PR 1969 Artificial feeding of infant rats by continuous gastric infusion. J Nutr 98:404-410 
21. Abbott FV, Ocvirk R, Najafee R, Franklin KB 1999 Improving the efficiency of the formalin test. Pain 83:561-569

22. Dickinson AL, Leach MC, Flecknell PA 2008 Influence of early neonatal experience on nociceptive responses and analgesic effects in rats. Lab Anim 43:11-16

23. Lidow MS, Song ZM, Ren K 2001 Long-term effects of short-lasting early local inflammatory insult. Neuroreport 12:399-403

24. Zahr LK, Balian S 1995 Responses of premature infants to routine nursing interventions and noise in the NICU. Nurs Res 44:179-185

25. Teng CJ, Abbott FV 1998 The formalin test: a dose-response analysis at three developmental stages. Pain 76:337-347

26. Anand KJ, Garg S, Rovnaghi CR, Narsinghani U, Bhutta AT, Hall RW 2007 Ketamine reduces the cell death following inflammatory pain in newborn rat brain. Pediatr Res 62:283-290
27. Anand KJ, Carr DB 1989 The neuroanatomy, neurophysiology, and neurochemistry of pain, stress, and analgesia in newborns and children. Pediatr Clin North Am 36:795-822

28. Bhutta AT, Anand KJ 2002 Vulnerability of the developing brain. Neuronal mechanisms. Clin Perinatol 29:357-372

29. Fitzgerald M 2005 The development of nociceptive circuits. Nat Rev Neurosci 6:507-520

30. Barr GA 1998 Maturation of the biphasic behavioral and heart rate response in the formalin test. Pharmacol Biochem Behav 60:329-335

31. Shair HN 2007 Acquisition and expression of a socially mediated separation response. Behav Brain Res 182:180-192

32. Walker CD, Kudreikis K, Sherrard A, Johnston CC 2003 Repeated neonatal pain influences maternal behavior, but not stress responsiveness in rat offspring. Brain Res Dev Brain Res 140:253-261 